Received: 11.06 .2017

Accepted: 18.11.2017

A - Study Design

B - Data Collection

C - Statistical Analysis

D - Data Interpretation

E - Manuscript Preparation

$F$ - Literature Search

$\mathrm{G}$ - Funds Collection

\section{Insight In Specific Phobias:}

\section{A FORGOTTEN BIAS}

Carlos Magalhães Coelho' ${ }^{1(A, B, D, E, F)}$, Kullaya Pisitsungkagarn ${ }^{1(\mathrm{~B}, \mathrm{E})}$, Nattasuda Taephant ${ }^{1(B, E)}$, Fernando Barbosa ${ }^{2(B, D, E)}$

${ }^{1}$ Faculty of Psychology, Chulalongkorn University, Bangkok, Thailand

${ }^{2}$ Laboratory of Neuropsychology, Faculty of Psychology and Education Sciences, Porto, Portugal

\title{
SUMMARY
}

The term insight is a major concept in psychiatry, which nonetheless has a number of different connotations ranging from awareness of one's disorder to achieving a clear and sudden solution of a problem or anosognosia. Although this concept is ubiquitous in the psychological literature, its definition is not clear, including in DSM-5; the most commonly used diagnostic system for mental disorders. This turns its use ambiguous, particularly for those who are not familiar with the historical background of the word. Here, we aim to a better understanding of insight in specific phobias and discuss the possible causes and implications in DSM- 5 criterion changes. The historical analysis of this concept lead to conclude three main assumptions: 1) There are probably different etiopathogenic mechanisms subjacent to the development of insight, anosognosia and possibly other "insight" related terms; 2 ) in the case of specific phobias, lack of insight is better seen and explained as a cognitive bias; 3) DSM use of an insight specifier for specific phobias could be of use; 4) lack of insight can be seen simply as a cognitive bias in most phobia cases.

Keywords: anosognosia, delusion, DSM 


\section{INTRODUCTION}

Historically, the evaluation of insight in specific phobias changed from more strict views in the DSM III (1980) claiming that a clear insight should be present, to later intermediate requirements (Beck, Emery, \& Greenberg, 2005) arguing that the person could know the disproportionate and excessiveness of their fear when they are distant or detached from it. In the most recent and quite opposite view, this requirement is no longer necessary.

Regarding other disorders, namely obsessive-compulsive disorder (OCD), body dysmorphic (BDD), and hoarding disorders, the DSM-5 has specifiers:

(1) good insight;

(2) poor insight;

(3) absent insight or delusional beliefs.

The main claim in this manuscript is that the lack of insight in specific phobias is an interpretive or appraisal type of bias. This bias can be acknowledged in the diagnostic as a specifier such as those previously described (good, poor, or absent insight). Considering insight in SP as a bias has significant advantages, as will be explained herein.

We first discuss the many uses of the term insight and its synonyms. We then outline the evolution of insight as a construct when applied to SP in the DSM. We conclude by discussing insight as a cognitive bias in SP and the implications of this view.

\section{The many uses of insight and the use of many terms meaning insight}

In the present edition of the Diagnostic and Statistical Manual of Mental Disorders(American Psychiatric Association, 2013), the disorders associated with limited insight include: delusional disorder, brief psychotic disorder, schizophrenia, schizoaffective disorder, bipolar disorders, social anxiety disorder, obsessive-compulsive disorder, hoarding disorder, body dysmorphic disorder, anorexia nervosa, substance-related disorders, neurocognitive disorders, and Huntington's disease. In personality changes due to another medical condition or strokerelated symptoms, insight is replaced by anosognosia, but with virtually the same meaning. For example: "Some individuals with psychosis may lack insight or awareness of their disorder (i.e., anosognosia) (p. 101)" following Babinski's (Babinski, 1914) use of the term.

As demonstrated above, the term insight is used in many disorders, but also interchangeably with awareness, denial, anosognosia, or self-consciousness (David, 1990; Markova \& Berrios, 1995), and may have many different meanings (Reddy, 2016). In the dictionary definition, insight is used as knowledge and referred to as a deep understanding (of a person or thing). When describing the grandiose type of delusional disorder, the DSM-5 gives as an example of a delusion theme the conviction of having some great (unrecognized) talent or insight, using the dictionary form. In addition, when insight is absent, it is termed delusional.

Among Gestalt psychologists, insight is seen as a sudden breakthrough of how to solve a problem (Bowden, Jung-Beeman, Fleck, \& Kounios, 2005). In 
psychoanalytic perspectives, insight is seen as a conscious awareness of wishes, defenses, and compromises that interact to produce emotional conflict or deficits in psychological development (Kivlighan Jr, Multon, \& Patton, 2000). Regarding psychosis, insight is the patients' recognition that they are suffering from a mental illness and their ability to re-attribute or re-label the experience of certain mental events as being pathological (David, 1990).

The lack of a precise definition creates difficulties in knowing insight's nature and role in different disorders (Marková \& Berrios, 2011). At present, the DSM carries a tremendous volume of information, but its clinical validity is becoming more often challenged (Mullins-Sweatt, Lengel, \& DeShong, 2016), partly due to conceptual overlapping and historical accretion and partly due to redefining syndromes without redefining signs and symptoms (Maj, 2016).

In fact, the use of different terminology can be required if there are different etiopathogenic causal mechanisms related to symptom awareness in various disorders. For example, anosognosia in hemiplegia is commonly associated with right hemisphere dysfunction (Heilman, 2014) and might be related to impaired sensory feedback (Frith, Blakemore, \& Wolpert, 2000). Thus, defining anosognosia, absent insight, and delusional beliefs as synonymous can be an invitation to misunderstandings. Adding the use of common everyday meanings of insight, such as factual insight and true insight (American Psychiatric Association, 2013) intermixed with different constructs sharing the same name and different names to describe the same construct, increases ambiguity, which will tend to become more convoluted as the volume of clinical and research data accumulates.

For the above reasons, the conceptual construction of insight seems without clinically unambiguous soundness. While for clinicians who use the DSM routinely for years it might seem quite evident, to the researcher or neuroscientist with no clinical experience, the said polysemic use of insight in the DSM and the use of interchangeable terms can be overwhelming. We next concisely explore the clinical and historical origins of the term insight in SP.

\section{DSM changes in insight regarding specific phobia}

Insight was an SP diagnostic criterion from 1980 (American Psychiatric Association, 1980) until its removal in 2013. Since the publication of the DSM-II in 1968 (American Psychiatric Association, 1968), insight had been seen as one of the main differential characteristics of neuroses. In fact, insight was the primary differential criterion between neuroses and psychoses, mainly because the former patients are not as detached from reality (Maser, Kaelber, \& Weise, 1991). The subsequent DSM formats kept the dichotomous distinction neuroses/psychoses by requiring adults to recognize their fear as excessive or irrational. Neurosis was seen as a condition in which the awareness of a patient's own disrupted or degraded mental functioning was present, and this recognition was called insight. This concept was originally defined as an all-or-nothing unitary phenomenon. Individuals were considered categorically to own it or lack it, but this would change over time. 
Different names were given since the first edition of the DSM to what we call today specific phobia, namely phobic reaction in 1952 by the time of the first DSM edition (American Psychiatric Association, 1952), phobic neurosis in the DSM-II (American Psychiatric Association, 1968), simple phobia in the DSM-III (American Psychiatric Association, 1980) and DSM-III-R (American Psychiatric Association, 1987), and finally specific phobia both in the DSM-IV (American Psychiatric Association, 1994) and DSM-IV-TR (American Psychiatric Association, 2000). The diagnosis of SP was characterized by an intense fear of an object or situation in which the individual had conscious awareness of it as not posing a real danger. In the DSM-III this was expressed in criterion B: “... recognition by the individual that his or her fear is excessive or unreasonable" (p. 230). Similarly, the 1987 DSM revised edition (American Psychiatric Association, 1987) required acknowledgment of the danger disproportionality. The absence of insight was seen as an important sign that other disorders should be considered, namely those of a psychotic nature.

Presently, insight is still often used as a descriptive feature specifier conveying supplementary information that can help design better treatment planning. Hence, the removal of insight criterion in SP in the DSM-5 does not reflect overall changes related to a diminished importance of insight since this concept was refined relatively in 3 other disorders. So, what where those reasons?

\section{Removing insight from SP}

One strong motivation for the DSM removal of Criterion $C$ (the person is able to assume that his or her fear is excessive or unreasonable) in SP was Zimmerman and colleagues' work (Zimmerman, Dalrymple, Chelminski, Young, \& Galione, 2010). The authors found that some patients with phobia were not diagnosed as phobic because they did not recognize the excessiveness or unreasonableness of their fear. As such, the Criterion $\mathrm{C}$ was creating a false-negative problem. Using a modified version of the Structured Clinical Interview for the DSM-IV, the authors interviewed 3,000 psychiatric outpatients and 1,800 candidates for bariatric surgery. Their analysis revealed that among the outpatients with SP, $4.1 \%$ (lifetime) and $4.2 \%$ (current diagnosis) did not meet Criterion C. This was considered a low percentage to justify keeping the criterion, in addition to creating the above-mentioned false-negative problem, since some patients that should be diagnosed with SP were excluded for not having met Criterion C.

Although these findings and arguments for the removal of insight as a criterion for SP seem sound, the non-inclusion of insight specifiers is surprising. Several arguments would favor removing Criterion $\mathrm{C}$ while still adding an insight specifier. First, it should be noted that OCD, one of the disorders that has the insight specifier, also presents $4 \%$ or fewer individuals with absent insight or delusional beliefs (American Psychiatric Association, 2013). Second, the removal of the insight criterion without the substitution of a similar specifier now used to classify OCD, $\mathrm{BDD}$, or HD does not acknowledge variability and within-category heterogeneity, potentially dropping inter-rater reliability ratings or favoring other diagnostics. 
Third, previous studies of phobic disorders have questioned the insight of patients with SP (Jones \& Menzies, 2000; Menzies \& Clarke, 1995; Menzies, Harris, \& Jones, 1998) and favored the inclusion of specifiers in SP.

In favor of our argument, a spider fear study from Jones and Menzies (Jones \& Menzies, 2000) found that even when removed from the proximity of the phobic stimulus, phobic subjects still gave higher estimates of the probability of injury resulting in harm and claimed more reasonability of their anxiety when compared to controls. Menzies and Clarke (1995) also compared danger perception of participants with acrophobia $(n=59)$ and a control group $(n=59)$. Evaluations were performed both before and during the exposure to a fire ladder attached to a parking lot. Before being examined, the participants with acrophobia estimated falling as more likely and the possible consequences of falling as being more damaging compared to the control group. They also believed that their levels of anticipatory anxiety were more reasonable and appropriate to the demands of the situation. Thus, their judgments and beliefs were excessive and unreasonable, defying the idea that participants with specific phobias have a clear insight regarding the inadequacy of their anxiety.

When these studies were performed, the DSM-IV (1994) had a subcategory of poor insight for obsessive-compulsive disorder in Axis V (Global Assessment of Functioning). Since the participants still scored very high regarding danger expectancies, even when removed from the fearful situation, this prompted Menzies and colleagues (1998) to propose a similar category of poor insight in specific phobias. In their study, 64 acrophobic participants were placed at the base of external stairs in a parking lot. From this situation, they were asked to predict the probability of falling if they went up the stairs. The results showed that the two groups could be separated based on a self-reported likelihood of falling superior or inferior to $50 \%$ : a good insight group $(n=31)$ and a poor insight group $(n=33)$. The good insight group rated the probability of falling at $9.84 \% \mathrm{com}$ pared to $88.6 \%$ in the poor insight group. As the groups were not different in age, gender, or average anxiety, the authors suggested that insight itself was the construct that best differentiated the groups.

As we have seen, the work of Menzies and colleagues suggests that insight may be poor even when the individual is distant from phobic cues. These reasons indicate that although Criterion C removal in SP might is justified, there are sound studies and arguments that indicate the importance of an insight specifier in SP.

\section{Insight as a cognitive bias in phobias}

Insight as self-knowledge into an individual's mental disorder can be a misleading definition when discerning phobias. Insight in SP is not about fear awareness, but rather about an excessive attribution of danger to a particular stimulus. The lack of insight regarding danger is an incorrect judgment and is based on the subjective appraisal of an external phenomenon (Moors, Ellsworth, Scherer, \& Frijda, 2013). Fear is an automatic system, involuntary and hard to access through introspection (Öhman \& Mineka, 2001), whereas insight is the evaluation 
of proportionality regarding the relationship between the stimuli and the dangers it might pose. This evaluation can suffer from an interpretive bias (MacLeod \& Mathews, 2012), which is known to exist in people with SP, and psychological explanations regarding fear posit attention and judgment biases as a cause of danger overestimation (Arntz, Rauner, \& Van den Hout, 1995).

Beck and colleagues (Beck, Baruch, Balter, Steer, \& Warman, 2004) termed cognitive insight as comprising "distorted beliefs and misinterpretations (p. 321)". A specific phobia is by standard definition a fear that is out of proportion to the danger, and the patient can be more or less aware of this (fear/danger) discrepancy. Therefore, insight in SP is the awareness of the gap lying between the perceived and the real danger. The insight regarding this disproportionality is a cognitive evaluation. As such, lack of insight is a cognitive bias.

Individuals with anxiety disorders more easily interpret information as indicative of a potential threat, creating a vicious cycle in which ambiguous situations are more often experienced as threatening (Beard, 2011; Hertel \& Mathews, 2011), so hypothetically changing this bias could change the feeling and behavior. However, in arachnophobia, for example, biases are common (Reese, McNally, Najmi, \& Amir, 2010), but cognitive bias modification (CBM) does not reduce spider fear (de Jong \& Muris, 2002). This result can be due to the fact that this procedure changes insight but not fear itself.

To summarize, in SP, poor insight can be of an interpretive nature and result from an appraisal bias. People with fear will tend to perceive exaggerated danger in case of ambiguity (for example, a dog may or may not be there, but I will go around anyway, just in case) or when information is not ambiguous (for example, the dog is small and friendly, but I stay far anyway because I feel bad). As Arntz and colleagues pointed out, participants with SP infer risk on the basis of their anxiety response (Arntz et al., 1995). An expectancy bias can exist regarding the possibility of imminent danger. However, the physical fear response to a phobic stimulus is more or less independent from the following cognitive meaning attributed by the participant (Coelho \& Purkis, 2009). That is, in SP, insight and bias seem to be independent from fear.

When the bias about the danger of the object or situation is extreme, it may impede phobic individuals from recognizing the irrationality of their beliefs regarding the threat of the stimuli, being in complete conviction of their beliefs. This justifies the use of the term delusion to indicate extreme lack of insight.

An insight specifier in specific phobias would stress the importance regarding the individual evaluation of the real danger of the feared object or situation. This is essential to treatment choices, as different levels of insight require different treatment approaches (Nemeroff et al., 2013). For example, using virtual reality (VR) technology, patients can become thoroughly immersed in a task. In a realistic VR environment, phobic individuals with a poor insight might be able to desensitize better in a first treatment approach, since there is no real danger (Coelho, Santos, Silvério, \& Silva, 2006). Conversely, phobic participants with good insight might 
be susceptible to less bias and hence more prone to other types of treatment, such as direct exposure therapy.

Conceptualizing insight in this perspective allows for a better evaluation and measurement of the bias (insight) in specific phobias and should lead to a better understanding and treatment of this condition, along with an improved interplay between the clinical and experimental studies of SP. It is important to remember that the clinician makes insight assessment. So, if clinicians can use experimental tools to measure insight, their biases in their clinical judgements will also be reduced.

\section{DISCUSSION}

Knowing the history of DSM changes regarding insight and its many meanings is useful for academics, clinicians, and researchers who focus on fear and phobias across various fields (for example, genetics, systems neuroscience, or behavioral science). Nonetheless, excessive historical burden given to classifying terms may contribute to a diagnostic classification resulting in historical accretion with little relevance to current scientific needs (Frances \& Widiger, 2012). In some cases, this method of preserving the history of clinical classification systems seems to be accumulating noise and hampering simplicity. Adding an insight descriptive specifier and defining the concept of insight can mitigate this cumulative conceptual historical burden.

Clarifying and agreeing on a practical terminology will improve both research and clinical practice. For insight to be defined using genetic, imaging, and neurochemical information (Helzer, Kraemer, \& Krueger, 2006), it needs to be anchored in an experimentally measurable way. Identifying insight as a measurable construct (bias) that has been studied in anxiety disorders for decades strongly approaches experimental and clinical psychopathology, creating bridges between these two fields that nomenclature now hinders.

Insight is also an important factor for predicting treatment. Although specific phobias can cause significant interference or distress and potentially impair occupational and interpersonal functioning, only a minority of affected persons seek professional help (Coles, Schubert, Heimberg, \& Weiss, 2014). This can be due to lack of insight, as people with low insight might not even consider treatment for their fear to seem reasonable. This is even more important since SP are one a common anxiety disorder with reported 12-month rates of $8.7 \%$ (Kessler et al., 2005).

The use of an insight specifier for specific phobias (for example, good, poor, or absent insight) seems appropriate in specific phobias, since a body of evidence shows that there are several levels of insight in this clinical condition. Defining insight as a bias also allows the clinician to better appraise patients' ability to evaluate the real danger of the stimulus, planning treatments more accurately, and predicting treatment adherence and compliance.

Regarding research, interpreting insight as a bias is useful not only to research itself, but also to facilitate communication with clinicians. For example, different subtypes of specific phobias might be more or less "vulnerable" to insight. It is also 
likely that appraisal and interpretive bias bears developmental origins leading to specific phobias. These include fear conditioning-based as well as vicarious and information learning as pathways leading to the development of specific phobias. Information learned fears might be more vulnerable to cognitive bias modification-1 (CBM-I) compared to traumatic fears. By the same token, prepared fears (that is, those associated with evolutionary fear-relevant stimuli) should be less susceptible to CBM-I. Therefore, many research opportunities can derive from observing insight as a bias, and insight can also be better understood as a bias with similar advantages in other disorders.

\section{ACKNOWLEDGEMENTS}

This research is supported by Rachadapisek Sompote Fund for Postdoctoral Fellowship, Chulalongkorn University, and a research funding from the faculty of Psychology division of research affairs of the same University, awarded to the first author. We would like to thank the anonymous referees and reviewers for their helpful comments in a previous version of this manuscript.

\section{Ethical approval}

This article does not contain any studies on human or animals participants performed by any of the authors. For this type of study formal consent is not required.

\section{REFERENCES}

American Psychiatric Association. (1952). Diagnostic and Statistical Manual of Mental Disorders. Washington, DC: American Psychiatric Press.

American Psychiatric Association. (1968). Diagnostic and Statistical Manual of Mental Disorders (2nd.ed. ed.). Washington, DC: American Psychiatric Press.

American Psychiatric Association. (1980). Diagnostic and Statistical Manual of Mental Disorders (3rd.ed. ed.). Washington, DC: American Psychiatric Press.

American Psychiatric Association. (1987). Diagnostic and Statistical Manual of Mental Disorders (3rd.ed., rev. ed.). Washington, DC: American Psychiatric Press.

American Psychiatric Association. (1994). Diagnosis and Statistical Manual of Mental Disorders (DSM-IV). Washington, DC: American Psychiatric Press.

American Psychiatric Association. (2000). DSM-IV-TR: Diagnostic and statistical manual of mental disorders, text revision. Washington, DC: American Psychiatric Press.

American Psychiatric Association. (2013). Diagnostic and Statistical Manual of Mental Disorders (DSM-5®). Wachington DC: American Psychiatric Press.

Arntz, A., Rauner, M., \& Van den Hout, M. (1995). "If I feel anxious, there must be danger": Ex-consequentia reasoning in inferring danger in anxiety disorders. Behaviour research and therapy, 33(8), 917-925.

Babinski, J. (1914). Contribution à l'étude des troubles mentaux dans l'hémiplégie organique cérébrale (anosognosie). Revue neurologique, 27, 845-848.

Beard, C. (2011). Cognitive bias modification for anxiety: current evidence and future directions. Expert review of neurotherapeutics, 11(2), 299-311.

Beck, A. T., Baruch, E., Balter, J. M., Steer, R. A., \& Warman, D. M. (2004). A new instrument for measuring insight: the Beck Cognitive Insight Scale. Schizophrenia research, 68(2), 319-329.

Beck, A. T., Emery, G., \& Greenberg, R. L. (2005). Anxiety disorders and phobias: A cognitive perspective: Basic Books. 
Bowden, E. M., Jung-Beeman, M., Fleck, J., \& Kounios, J. (2005). New approaches to demystifying insight. Trends in cognitive sciences, 9(7), 322-328.

Coelho, C. M., \& Purkis, H. (2009). The origins of specific phobias: Influential theories and current perspectives. Review of General Psychology, 13(4), 335.

Coelho, C. M., Santos, J. A., Silvério, J., \& Silva, C. F. (2006). Virtual reality and acrophobia: oneyear follow-up and case study. CyberPsychology \& Behavior, 9(3), 336-341.

Coles, M. E., Schubert, J. R., Heimberg, R. G., \& Weiss, B. D. (2014). Disseminating treatment for anxiety disorders: step 1: recognizing the problem as a precursor to seeking help. Journal of Anxiety Disorders, 28(8), 737-740.

David, A. S. (1990). On insight and psychosis: discussion paper. Journal of the Royal Society of Medicine, 83(5), 325-329.

de Jong, P. J., \& Muris, P. (2002). Spider phobia: Interaction of disgust and perceived likelihood of involuntary physical contact. Journal of Anxiety Disorders, 16(1), 51-65.

Frances, A. J., \& Widiger, T. (2012). Psychiatric diagnosis: lessons from the DSM-IV past and cautions for the DSM-5 future. Annual Review of Clinical Psychology, 8, 109-130.

Frith, C. D., Blakemore, S.-J., \& Wolpert, D. M. (2000). Abnormalities in the awareness and control of action. Phil. Trans. R. Soc. Lond. B, 355(1404), 1771-1788.

Heilman, K. M. (2014). Possible mechanisms of anosognosia of hemiplegia. Cortex, 61, 30-42. Hiatric diagnoses. Psychological Medicine, 36(12), 1671-1680.

Hertel, P. T., \& Mathews, A. (2011). Cognitive bias modification: Past perspectives, current findings, and future applications. Perspectives on Psychological Science, 6(6), 521-536.

Jones, M. K., \& Menzies, R. G. (2000). Danger expectancies, self-efficacy and insight in spider phobia. Behaviour research and therapy, 38(6), 585-600.

Kessler, R. C., Berglund, P., Demler, O., Jin, R., Merikangas, K. R., \& Walters, E. E. (2005). Lifetime prevalence and age-of-onset distributions of DSM-IV disorders in the National Comorbidity Survey Replication. Archives of general psychiatry, 62(6), 593-602.

Kivlighan Jr, D. M., Multon, K. D., \& Patton, M. J. (2000). Insight and symptom reduction in timelimited psychoanalytic counseling. Journal of Counseling Psychology, 47(1), 50.

MacLeod, C., \& Mathews, A. (2012). Cognitive bias modification approaches to anxiety. Annual Review of Clinical Psychology, 8, 189-217.

Maj, M. (2016). Narrowing the gap between ICD/DSM and RDoC constructs: possible steps and caveats. World Psychiatry, 15(3), 193-194.

Markova, I., \& Berrios, G. (1995). Insight in clinical psychiatry revisited. Comprehensive psychiatry, 36(5), 367-376.

Marková, I. S., \& Berrios, G. E. (2011). Awareness and insight in psychopathology: An essential distinction? Theory \& Psychology, 21(4), 421-437.

Maser, J. D., Kaelber, C., \& Weise, R. E. (1991). International use and attitudes toward DSM-III and DSM-III-R: Growing consensus in psychiatric classification. Journal of Abnormal Psychology, 100(3), 271.

Menzies, R. G., \& Clarke, J. C. (1995). Danger expectancies and insight in acrophobia. Behaviour research and therapy, 33(2), 215-221.

Menzies, R. G., Harris, L. M., \& Jones, M. K. (1998). Evidence from three fearful samples for a poor insight type in specific phobia. Depression and anxiety, 8(1), 29-32.

Moors, A., Ellsworth, P. C., Scherer, K. R., \& Frijda, N. H. (2013). Appraisal theories of emotion: State of the art and future development. Emotion Review, 5(2), 119-124.

Mullins-Sweatt, S. N., Lengel, G. J., \& DeShong, H. L. (2016). The importance of considering clinical utility in the construction of a diagnostic manual. Annual Review of Clinical Psychology, 12, 133-155.

Nemeroff, C. B., Weinberger, D., Rutter, M., MacMillan, H. L., Bryant, R. A., Wessely, S., . . Berk, M. (2013). DSM-5: a collection of psychiatrist views on the changes, controversies, and future directions. BMC medicine, 11(1), 202.

Öhman, A., \& Mineka, S. (2001). Fears, phobias, and preparedness: toward an evolved module of fear and fear learning. Psychological review, 108(3), 483. 
Reddy, M. (2016). Lack of insight in psychiatric illness: A critical appraisal. Indian journal of psychological medicine, 38(3), 169.

Reese, H. E., McNally, R. J., Najmi, S., \& Amir, N. (2010). Attention training for reducing spider fear in spider-fearful individuals. Journal of Anxiety Disorders, 24(7), 657-662.

Zimmerman, M., Dalrymple, K., Chelminski, I., Young, D., \& Galione, J. N. (2010). Recognition of irrationality of fear and the diagnosis of social anxiety disorder and specific phobia in adults: Implications for criteria revision in DSM-5. Depression and anxiety, 27(11), 1044-1049.

\section{Corresponding author}

Coelho C.M

Faculty of Psychology,

Chulalongkorn University, Bangkok, Thailand

e-mail: carlos.s@chula.ac.th 